\title{
EDUCACIÓN PRENATAL Y SU RELACIÓN CON EL TIPO DE PARTO: UNA VÍA HACIA EL PARTO NATURAL
}

\author{
Carmen Soto L. ${ }^{a}$, Hilda Teuber L. ${ }^{a}$, Clemencia Cabrera F.a, Miguel Marín N. ${ }^{1}$, Jorge Cabrera \\ D. ${ }^{2}$, Mahal Da Costa S. ${ }^{b}$, Heriberto Araneda C. ${ }^{c}$ PhD \\ ${ }^{1}$ Servicio de Obstetricia y Ginecología, Hospital Guillermo Grant Benavente, Concepción. ${ }^{2}$ Departamento de Obstetricia \\ y Ginecología, Facultad de Medicina, Universidad de Concepción.
}

a Matrona, Departamento de Obstetricia y Ginecología, Facultad de Medicina, Universidad de Concepción. ${ }^{b}$ Profesor de

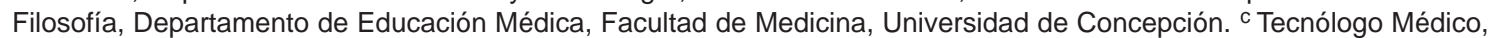
Departamento de Obstetricia y Ginecología, Facultad de Medicina, Universidad de Concepción.

\begin{abstract}
RESUMEN
Objetivo: Aplicar una intervención educativa durante la gestación y observar los efectos sobre el embarazo, parto y recién nacido. Método: Se realizó un estudio con diseño semi experimental de serie de casos con 40 primíparas normales, desde las 20 semanas de gestación en dos Centros de Salud Municipales de Concepción, Chile, desde 2001 a 2004. Se aplicó la intervención educativa en 8 sesiones a las gestantes con su pareja y doula. Los resultados se compararon con un grupo de referencia de 55 primíparas normales. Resultados: La tasa de cesárea fue de 7,5\% en el grupo estudio y $27,3 \%$ en el grupo de referencia $(p<0,01)$; hubo patología del embarazo en $17,5 \%$ versus $47,3 \%(p<0,05)$; uso de ocitocina en $40 \%$ versus $78,2 \%(p<0,01)$; episiotomía en $64,9 \%$ versus $100 \%(p<0,01)$. El Apgar $<7$ al primer minuto fue $5 \%$ grupo estudio y $5,4 \%$ grupo referencia, diferencia no significativa. Conclusiones: La educación en el control prenatal utilizando protocolos, reduce el número de intervenciones rutinarias e innecesarias durante el embarazo y parto.
\end{abstract}

PALABRAS CLAVES: Educación prenatal, apego, parto natural, doula

\section{SUMMARY}

Objective: To determine that an educative intervention during pregnancy has effects on the pregnancy, delivery and newborn. Method: A semi experimental study design was made in series of normal cases with 940 primiparous, since 20 weeks of gestation in two Municipal Centers of Health, Concepción, Chile, between 2001 and 2004. It was applied an educative intervention in 8 sessions to 40 primiparous with his respective partner and doula. The results were compared with a reference group of 55 normal primiparous. Results: The comparison between the study and reference groups show significant difference in: pregnancy pathology, $17.5 \%$ versus $47.3 \%$ ( $p<0.05)$; use of oxytocin, $40 \%$ versus $78.2 \%(p<0.01)$; need of episiotomy, $64.9 \%$ versus $100 \%(p<0.01)$; caesarean section rate, $7.5 \%$ versus $27.3 \%(p<0.01)$. Conclusion: Education during gestation using protocols in the prenatal control reduced the number of unnecessary routine intervention during pregnancy and birth.

KEY WORDS: Prenatal education, bonding, natural birth, doula 


\section{INTRODUCCIÓN}

En el último tiempo se ha observado un aumento de publicaciones científicas que enfatizan la promoción y práctica del parto natural $(1,2,3,4)$. Esta tendencia surge como cuestionamiento frente a la excesiva tasa de cesáreas (5) y de intervenciones médicas de rutina durante el trabajo de parto y parto tales como el uso de ocitocina, monitorización electrónica fetal, rotura artificial de las membranas ovulares, enema evacuante y episiotomía en primíparas $(6,7)$.

El aumento en las tasas de cesárea a nivel mundial (8), ha motivado a investigadores e instituciones de salud a proponer estrategias para lograr su reducción, entre ellas protocolizar las indicaciones de cesárea, pedir una segunda opinión a pares y hacer públicas las tasas de cesáreas (9). En Chile la tasa de cesárea en 2004 fue $31,7 \%$ y $33,4 \%$ en el Hospital Guillermo Grant Benavente de Concepción, cifras que sobrepasan lo recomendado por la OMS (10).

La cesárea es una intervención quirúrgica imprescindible cuando la madre o el feto están en riesgo. El avance en las técnicas quirúrgicas, el advenimiento de los antibióticos y de los cuidados anestésicos, han minimizado sus complicaciones. Sin embargo, la cesárea tiene 2 a 3 veces mayor tasa de mortalidad materna, mayor tasa de infección, mayor costo que un parto normal y mayor trauma para el neonato, entre otros (11). La principal causa de cesárea es la cesárea anterior (12), por tanto las estrategias están dirigidas a evitar la primera cesárea.

El objetivo de este estudio fue determinar si la aplicación de una intervención educativa realizada durante el embarazo, tiene efectos observables sobre la evolución del embarazo, parto y recién nacido.

\section{METODOLOGÍA}

Se realizó un estudio con diseño semi experimental, de serie de casos en los Centros de Salud San Pedro de la Paz y Boca Sur de la comuna de Concepción, entre los años 2001 a 2004. Previo consentimiento informado, se invitó a participar a 60 gestantes nulíparas de bajo riesgo a las 20 semanas de gestación; 40 aceptaron, las que constituyeron el grupo estudio. Se eligió una muestra de 55 nulíparas que tuvieron su parto en enero de 2000, las que formaron el grupo de referencia. El criterio de inclusión en ambos grupos fue primíparas sin patología asociada. Esta investiga- ción fue aprobada por el comité de ética de la Facultad de Medicina de la Universidad de Concepción.

La intervención educativa se denominó "Renacer del Parto Natural" y se aplicó en 8 sesiones a partir de las 20 semanas de gestación. En esta investigación se entregaron herramientas y se desarrollaron habilidades para enfrentar el trabajo de parto en forma natural y protagónica, junto a su pareja y doula (persona de sexo femenino, elegida por la embarazada cuya función es ser acompañante en el curso, durante el proceso del embarazo, trabajo de parto y parto) (1).

Las variables dependientes fueron: cesárea, enema, inicio del parto, libertad de movimiento en el trabajo de parto, ocitocina, rotura artificial de las membranas ovulares, monitorización electrónica fetal, posición de la madre en el período expulsivo, episiotomía, tiempo de apego del recién nacido con su madre y la variable independiente fue el programa educativo.

Los datos se analizaron con la prueba de chi cuadrado y la prueba t de Student, según correspondiera. Se consideró significativo un $\mathrm{p}<0,05$.

\section{RESULTADOS}

Las características demográficas de los grupos estudio y referencia se presentan en la Tabla I; no hubo diferencias significativas en las variables analizadas. El embarazo evolucionó sin patología en el $82,5 \%$ de las gestantes del grupo estudio contra un $52,7 \%,(p<0,05)$ del grupo de referencia (Tabla II). En las características del trabajo de parto solo hubo diferencias significativas en el mayor uso de ocitocina $(p<0,01)$ y de enema $(p<0,05)$, como menor movilidad en trabajo de parto en el grupo de referencia $(p<0,01)$ (Tabla III). En el grupo estudio el $7,5 \%$ tuvo cesárea y $27,3 \%$ en el de referencia $(p<0,01)$; el grupo estudio presentó parto natural en el $20 \%$ versus $0 \%$ en el grupo de referencia; episiotomía se realizó en $64,9 \%$ del grupo estudio versus $100 \%$ en el grupo referencia $(p<0,01)$; posición libre en el expulsivo la utilizó el $27,7 \%$ de las gestantes del grupo estudio versus $0 \%$ grupo referencia $(p<0,01)$ (Tabla IV). Tuvo acompañamiento continuo el $60 \%$ de las mujeres del grupo estudio versus $1,8 \%$ del grupo de referencia $(p<0,01)$ (Tabla $V)$. No hubo diferencias significativas entre las variables neonatales analizadas; el tiempo promedio de apego en el grupo estudio fue de 17 minutos (Tabla VI). La Tabla VII muestra el tipo de patología en ambos grupos observándose diferencias significa- 
Tabla I

CARACTERÍSTICAS DE LAS EMBARAZADAS EN ESTUDIO

\begin{tabular}{lccc}
\hline Variable & $\begin{array}{c}\text { Grupo estudio } \\
n=40\end{array}$ & $\begin{array}{c}\text { Grupo referencia } \\
n=55\end{array}$ & Valor $p$ \\
\hline Edad (años) & $21,8 \pm 4,9$ & $21,6 \pm 5,1$ & NS \\
Escolaridad (años) & $11,2 \pm 2,4$ & $10,3 \pm 2,5$ & NS \\
Nivel educacional & & & NS \\
$\quad$ Educación básica & $9(22,5 \%)$ & $14(25,5 \%)$ & NS \\
$\quad$ Media & $22(55,0 \%)$ & $38(69,1 \%)$ & NS \\
$\quad$ Superior & $9(22,5 \%)$ & $3(5,4 \%)$ & NS \\
Estado civil & $14(21,3 \%)$ & $20(36,4 \%)$ & NS \\
$\quad$ Casada & $21(55,3 \%)$ & $27(49,1 \%)$ & NS \\
$\quad$ Soltera & $5(23,4 \%)$ & $8(14,5 \%)$ & NS \\
$\quad$ Conviviente & $16(40 \%)$ & $35(63,6 \%)$ & NS \\
Actividad & $14(35 \%)$ & $8(14,5 \%)$ & NS \\
$\quad$ Dueña de casa & $4(10 \%)$ & $5(9,1 \%)$ & NS \\
$\quad$ Estudiante & $6(15 \%)$ & $7(12,8 \%)$ & \\
$\quad$ Actividades del hogar & & & \\
$\quad$ Otras & & & \\
\hline
\end{tabular}

NS: no significativo.

Tabla II

CARACTERÍSTICAS DE LA EVOLUCIÓN DEL EMBARAZO

\begin{tabular}{lccc}
\hline Evolución del embarazo & $\begin{array}{c}\text { Grupo estudio } \\
n(\%)\end{array}$ & $\begin{array}{c}\text { Grupo referencia } \\
n(\%)\end{array}$ & Valor $p$ \\
\hline Sin patología & $33(82,5)$ & $29(52,7)$ & $<0,05$ \\
Con Patología & $7(17,5)$ & $26(47,3)$ & $55(100)$ \\
\hline Total & $40(100)$ & 5 & \\
\hline
\end{tabular}

Tabla III

CARACTERÍSTICAS DEL TRABAJO DE PARTO

\begin{tabular}{|c|c|c|c|c|c|}
\hline \multirow{2}{*}{$\begin{array}{l}\text { Variable } \\
\text { Inicio del parto }\end{array}$} & \multicolumn{2}{|c|}{$\begin{array}{c}\text { Grupo estudio } \\
n=40\end{array}$} & \multicolumn{2}{|c|}{$\begin{array}{c}\text { Grupo referencia } \\
\qquad n=55\end{array}$} & \multirow[t]{2}{*}{ Valor $p$} \\
\hline & $n$ & $\%$ & $n$ & $\%$ & \\
\hline Espontáneo & 32 & 80 & 31 & 56,4 & NS \\
\hline Inducido y cesárea & 8 & 20 & 24 & 43,6 & \\
\hline Uso ocitocina & 16 & 40 & 43 & 78,2 & $<0,01$ \\
\hline Enema & 17 & 42,5 & 51 & 92,7 & $<0,05$ \\
\hline \multicolumn{6}{|l|}{ Rotura Artificial de Membranas (RAM) } \\
\hline Con RAM & 22 & 55 & 42 & 76,4 & NS \\
\hline \multicolumn{6}{|l|}{ Libertad de movimiento en trabajo de parto } \\
\hline Libre posición & 19 & 48,7 & 2 & 4 & 0,01 \\
\hline
\end{tabular}

NS: no significativo.

tivas en la presencia de los síndromes hipertensivos del embarazo, 5\% grupo estudio versus $53,8 \%$ grupo referencia. La Tabla VIII muestra el tipo de parto según evolución embarazo, obser- vándose en el grupo estudio $40 \%$ de cesárea versus $53 \%$ en el grupo de referencia, diferencia no significativa. La Tabla IX muestra las causas de cesárea en ambos grupos. 
Tabla IV

CARACTERÍSTICAS DEL PARTO

\begin{tabular}{lccccc}
\hline & Grupo estudio $(n=40)$ & Grupo referencia $(n=55)$ & Valor $p$ \\
\hline Tipo de parto & $n$ & $\%$ & $n$ & $\%$ & $<0,01$ \\
$\quad$ Normal & 29 & 72,5 & 40 & 72,7 & 0 \\
$\quad$ Natural & 8 & 20,0 & 0 & 27,3 & $<0,01$ \\
$\quad$ Cesárea & 3 & 7,5 & 15 & 100,0 & $<0,05$ \\
$\begin{array}{l}\text { Episiotomía } \\
\quad \text { Con episiotomía }\end{array}$ & 24 & 64,9 & 40 & & 0 \\
$\begin{array}{l}\text { Posición en el parto } \\
\text { Libre }\end{array}$ & 10 & 27,1 & 0 & & \\
\hline
\end{tabular}

Tabla V

ACOMPAÑAMIENTO CONTINUO EN TRABAJO

DE PARTO Y PARTO

\begin{tabular}{lccccc}
\hline & Grupo estudio $(n=40)$ & Grupo referencia $(n=55)$ & Valor $p$ \\
\hline Compañía & $n$ & $\%$ & $n$ & $\%$ & $<0,01$ \\
Con compañía & 24 & 60 & 1 & 1,8 & \\
Tipo de compañía & & & & & \\
Pareja & 6 & 25 & 0 & 0 \\
Doula & 6 & 25 & 0 & 0 \\
Pareja y doula & 12 & 50 & 0 & 0 \\
\hline
\end{tabular}

Tabla VI

RESULTADOS NEONATALES

\begin{tabular}{lccc}
\hline Variable & $\begin{array}{c}\text { Grupo estudio } \\
n(\%)\end{array}$ & $\begin{array}{c}\text { Grupo referencia } \\
n(\%)\end{array}$ & Valor $p$ \\
\hline Apgar & & & $52(94,5)$ \\
Apgar $\geq 7$ al 1er minuto & $38(95)$ & $3(5,5)$ & NS \\
Apgar $<7$ al 1er minuto & $2(5)$ & - & - \\
Apgar $<7$ a los 5 minutos & - & NR & NS \\
Promedio de apego (minutos) & 17 & $3.368,1 \pm 415,1$ & Peso promedio RN (gramos) \\
Pem,
\end{tabular}

NR: no reportado.

NS: no significativo.

\section{DISCUSIÓN}

Un número creciente de evidencias en la literatura en Chile sugieren hacer esfuerzos con el objeto de disminuir las tasas de cesáreas y de intervenciones médicas de rutina innecesarias durante el trabajo de parto y parto $(13,14)$. Nuestro estudio reporta una tasa de $7,5 \%$ lo que significa una reducción de la tasa de cesárea a la mitad de lo sugerido por la OMS (15) y entre 4 a 5 veces menos que lo reportado a nivel regional y nacional. La patología más frecuente presentada durante el embarazo fue el síndrome hipertensivo del embarazo, sin embargo esta patología no fue determinante en el resultado del parto. En este estudio las causas que determinaron la cesárea responden a las mismas causas que reporta la literatura (12). 
Tabla VII

DISTRIBUCIÓN DE PATOLOGÍAS Y EMBARAZO

\begin{tabular}{lrrrrr}
\hline Patologías & \multicolumn{2}{c}{ Grupo estudio } \\
$n=40$ & \multicolumn{2}{c}{$\begin{array}{c}\text { Grupo referencia } \\
n=55\end{array}$} & Valor $p$ \\
\hline Síndrome hipertensivo & $n$ & $\%$ & $n$ & $\%$ \\
Colestasia intrahepática & 2 & 5,0 & 14 & 25,0 & $<0,01$ \\
Síntoma parto prematuro & 1 & 2,5 & 1 & 1,9 & NS \\
Oligohidramnios & 0 & 0 & 1 & 1,9 & NS \\
Polihidramnios & 1 & 2,5 & 2 & 3,7 & NS \\
Diabetes gestacional & 0 & 0 & 1 & 1,9 & NS \\
Pielonefritis aguda & 0 & 0 & 2 & 3,7 & NS \\
Restricción crecimiento fetal & 0 & 0 & 3 & 5,5 & NS \\
\hline Total & 3 & 7,5 & 2 & 3,7 & NS \\
\hline
\end{tabular}

$\mathrm{NS}=$ No significativo.

Por otra parte, la episiotomía es una intervención utilizada rutinariamente en primíparas en la maternidad del hospital. Nuestro estudio muestra que la episiotomía se realizó en $64,9 \%$, lo que contrasta con las publicaciones de Carroli y Belizán publicada en la Base datos Cochrane sobre una revisión sistemática en el que se incluyeron seis estudios y cuyos resultados fueron una tasa de $27,6 \%$ de episiotomía en el grupo de episiotomía restrictiva (16). A pesar que en esta investigación se diseñaron protocolos tanto para la atención del trabajo de parto y parto en el que se destacaba el uso restrictivo de episiotomía, este estudio muestra una alta tasa de este procedimiento, pero, que comparado con el grupo histórico del Hospital Guillermo Grant Benavente la diferencia es significativa.

Los protocolos para la atención del parto enfatizan sobre la libertad de posición en el período expulsivo, en especial la posición vertical. Esta posición, tiene ventajas por sobre la posición de litotomía, como tasas más bajas de cesárea,

Tabla VIII

TIPO DE PARTO Y PATOLOGÍA DEL EMBARAZO

\begin{tabular}{lccccc}
\hline Causa & $\begin{array}{c}\text { Grupo } \\
\text { estudio }\end{array}$ & \multicolumn{2}{c}{$\begin{array}{c}\text { Grupo } \\
\text { referencia }\end{array}$} & Valor $p$ \\
& $n=7$ & $\%$ & \multicolumn{2}{c}{$n=26$} & $\%$
\end{tabular}

$\mathrm{NS}=$ No significativo. episiotomía, analgesia, ocitocina y duración del período expulsivo. Nuestro estudio reportó que sólo $27 \%$ de las gestantes tuvieron la opción de usar posición libre en este período, que en comparación con los reportes del Hospital Guillermo Grant Benavente es una diferencia significativa. Sin embargo, está bajo las recomendaciones de la OMS que sugiere que todas las mujeres que tienen parto vaginal no deben utilizar la posición de litotomía y concuerdan con lo reportado por Gupta y Hofmeyr (17).

El acompañamiento de la gestante durante el embarazo, trabajo de parto y parto por la doula y pareja fue una de las propuestas más importantes de la investigación (18). Los resultados de nuestro estudio coinciden con los reportados, que señalan que aquellas mujeres que tuvieron apoyo continuo durante el parto tienen resultados significativos en términos de reducción en la tasa de cesárea, partos instrumentalizados, menos anestesia, episiotomía, uso de ocitocina y mayor tiempo de apego $(8,18-25)$.
Tabla IX

CAUSAS DE CESÁREA

\begin{tabular}{lcc}
\hline Causa & $\begin{array}{c}\text { Grupo } \\
\text { estudio }\end{array}$ & $\begin{array}{c}\text { Grupo } \\
\text { referencia }\end{array}$ \\
\hline Desproporción céfalo-pélvica & 1 & 4 \\
Sufrimiento fetal agudo & 0 & 4 \\
Presentación podálica & 1 & 2 \\
Indicación médica & 1 & 5 \\
\hline Total & 3 & 15 \\
\hline
\end{tabular}




\section{CONCLUSIONES}

La educación en el embarazo más la protocolización de la atención del trabajo y parto redujo las intervenciones de rutina usadas en este proceso, lográndose un $20 \%$ de parto natural y $7,5 \%$ de cesáreas en el grupo intervenido.

\section{BIBLIOGRAFÍA}

1. Langer A, Campero L, García C, Reynoso S. Effects of psychosocial support during labor and childbirth on breastfeeding, medical interventions, and mother's wellbeing in a Mexican public hospital: a randomized clinical trial. Br J Obstet Gynaecol 1998; 105: 105663.

2. Murray Susan. Relation between private health insurance and high rates of cesarean section in Chile: Qualitative and quantitative study. BMJ 2000; 321: 1501-08.

3. Torres J, Kopplin E, Peña V, Klaus M, Salinas R, Herrera M. Impacto del apoyo emocional durante el parto en la disminución de cesáreas y gratificación del proceso. Rev Chil Obstet Ginecol 1999; 64(5): 405-12.

4. Walling A. Do doulas have a positive effect on outcome of labor? Am Family Physician 1999; 59: 2870-1.

5. White M. Obstetrics procedures and cesarean delivery rates continue to rise in North America. Int J Childbirth Educ 2004; 19(2): 16-7.

6. Belizán J, Althabe F, Barros F, Alexander S. Rates and implications of caesarean sections in Latin America: Ecological study. BMJ 1999; 319: 1397402.

7. Besio M. Cesárea versus parto vaginal. Una perspectiva ética. Rev Med Chile 1999; 127: 1121-5.

8. Walsh D. Subverting the assembly-line: Childbirth in a free-standing Birth centre. Social Science \& Medicine. In press, 2005.

9. Althabe F, Belizán J, Alexander S, et al. Mandatory second opinion to reduce rates of unnecessary caesarean sections in Latin America: a cluster randomized controlled trial. Lancet 2004; 363: 193440.

10. Ministerio de Salud. Estadísticas Nacionales y Servicio de Hospital Guillermo Grant Benavente, Concepción, 2004.

11. Shin K, Brubaker K, Ackerson L. Risk of cesarean delivery in nulliparous woman at greater than 41 weeks' gestational age with an unengaged vertex. Am J Obstet Gynecol 2004; 190: 129-34.

12. Lee I. Operación cesárea: estudio de causas y tendencias en un hospital de segundo nivel. Rev Med IMSS 2004; 42(3): 199-04.
13. Salinas H; Carmona S; Albornoz $\mathrm{J}$ et al. ¿Se puede reducir el índice de cesárea? Experiencia del Hospital Clínico de la Universidad de Chile. REV CHIL OBstet Ginecol 2004; 69(1): 8-13.

14. Cabero L. Estrategias para disminuir las tasas de cesárea. Disponible en: http://www.sego.es/ index.php?option=com_content\&task=view\&id=26\&ltemid=20

15. Organización Mundial de la Salud. Declaración de Fortaleza. Tecnología apropiada para el parto. Lancet 1985; 2: 436-7.

16. Carroli G, Belizán J. Episiotomía en el parto vaginal (Revisión Cochrane traducida). En: La Biblioteca Cochrane Plus, número 2, 2005. Oxford, Update Software Ltda. Disponible: http://www.updatesoftware.com.

17. Gupta JK, Hofmeyr GJ. Posición de la mujer durante el período expulsivo del trabajo de parto (Revisión Cochrane traducida). En: La Biblioteca Cochrane Plus, número 4, 2005. Oxford, Update Software Ltda. Disponible en: http://www.updatesoftware.com

18. Hodnett ED. Apoyo de prestadores de salud a la mujer durante el trabajo de parto. Biblioteca de Salud Reproductiva. OMS, 3, 2000.

19. Ministerio de Salud, Universidad de Chile. Nacer en el Siglo XXI, de vuelta a lo humano. Humanización del Proceso Reproductivo Mujer-Niño-Familia, Santiago, 2001.

20. Ministerio de Salud. Programa de Salud de la Mujer. Chile, 1997.

21. Scout K, Berkowitz G, Klaus M. A comparison of intermittent and continuos support during labor: $A$ meta-analysis. Am J Obstet Gynecol 1999; 180: 1054-9.

22. Uribe C, Poupin L, González R, Rodríguez J. Protagonismo de la embarazada durante su trabajo de parto. Efectos sobre los resultados maternos perinatales. Rev Chil Obstet Ginecol 2000; 65(5): 371-7.

23. Valdés V, Morlans X. Aporte de las doulas a la obstetricia moderna. Rev Chil Obstet Ginecol 2005; 70(2): 108-12.

24. Zhang J, Bernasko JW, Leybovich E, Fahs M, Hatch M. Continuos labor support from labor attendant for primiparous women: A meta-analysis. Obst Gynecol 1996; 88(4): 739-44.

25. Chalmers B, Mangiaterra V, Porter R. WHO principles of perinatal care: the essential antenatal, perinatal, and postpartum care course. Birth 2001; 28: 202-7.

Proyecto financiado por la Dirección de Investigación de la Universidad de Concepción, N²02.84B010-1.0 